\title{
Dexmedetomidina genera óxido nítrico mediante un mecanismo independiente de la óxido nítrico sintasa inducible
}

\author{
Jaime Riquelme ${ }^{1}$, Francisco Westermeier ${ }^{1}$, Zully Pedrozo ${ }^{1-3}$, Lorena García ${ }^{1}$, \\ Mauricio Ibacache ${ }^{4}$, Gina Sánchez ${ }^{3 *}$, Sergio Lavandero ${ }^{1-3-5^{*}}$ \\ 1 Advanced Center for Chronic Disease (ACCDiS), Facultad de Ciencias Químicas y \\ Farmacéuticas \& Facultad de Medicina, Universidad de Chile, Santiago, Chile. \\ 2 The Hatter Cardiovascular Institute, University College London, London, United Kingdom. \\ 3 Instituto de Ciencias Biomédicas (ICBM), Facultad de Medicina, Universidad de Chile, \\ Santiago, Chile. \\ 4 División de Anestesiología. Facultad Medicina, Pontificia Universidad Católica de Chile, \\ Santiago, Chile. \\ 5 Department of Internal Medicine (Cardiology Division), University of Texas Southwestern \\ Medical Center, Dallas, Texas, USA.
}

El infarto del miocardio es una de las principales causas de muerte a nivel mundial y se produce a consecuencia de procesos de isquemia-reperfusión (IR). El daño miocárdico generado por IR puede ser atenuado a través del pre-condicionamiento isquémico (PI) temprano, mediado por la vía RISK o PI tardío, que se asocia a una respuesta genómica en la que se activan proteínas como óxido nítrico sintasa inducible (iNOS).

Las vías de señalización que median el PI también pueden ser activadas farmacológicamente. Dexmedetomidina (Dex) es un agonista alfa2-adrenérgico, que se ha descrito como un potente agente cardioprotector frente a IR. Recientemente, nuestro grupo describió que Dex requiere el endotelio y la activación de la vía óxido nítrico sintasa endotelial (eNOS)-óxido nítrico (NO) para pre-condicionar el miocardio. Sin embargo, no existen estudios que muestren la posible participación de iNOS en la protección conferida por Dex.

La presente adenda tiene por objetivo evaluar si Dex activa iNOS en el corazón y en cardiomiocitos. Para esto, corazones de rata adulta fueron estimulados con Dex $10 \mathrm{nM}$ y se observó que el fármaco aumentó la producción de NO medida por cuantificación de nitritos, mas no estimuló la activación de iNOS medida por Western blot. Además, Dex tampoco indujo el aumento de mRNA de iNOS en cardiomiocitos adultos. Por lo tanto, Dex genera NO independiente a iNOS durante su efecto pre-condicionante agudo. Sin embargo, se requieren más estudios que clarifiquen su papel en una posible protección a largo plazo frente a IR generada por Dex.

Palabras clave: Dexmedetomidina, Corazón, iNOS, Pre-condicionamiento, Isquemia-reperfusión.

Correspondencia:

Gina Sánchez

Facultad de Medicina, Universidad de Chile.

gisanchez@med.uchile.cl

Sergio Lavandero

Facultad Ciencias Químicas y Farmacéuticas, Universidad de Chile

slavander@uchile.cl 


\section{Dexmedetomidine protects the heart against ischemia-reperfusion injury by an endothelial eNOS/NO dependent mechanism.}

Myocardial infarction is one of the leading causes of death worldwide and is generated as a consequence of ischemia-reperfusion (IR). Myocardial damage inflicted by IR can be attenuate by early ischemic pre-conditioning (IP), which is mediated by the RISK pathway or late IP, which is associated to a genomic response involving the activation of proteins such as inducible nitric oxide synthase (iNOS). The signaling pathways mediating IP can also be pharmacologically activated. Dexmedetomidine (Dex) is an alpha2-adrenergic receptor agonist, which has been described as a strong cardio protective agent against IR. Recently, our group reported that Dex requires the endothelium and the activation of the endothelial nitric oxide synthase (eNOS)-nitric oxide (NO) pathway to precondition the myocar- dium. However, there are no studies showing the involvement of iNOS in the protection elicited by Dex. The aim of this Addendum is to evaluate if Dex activates iNOS in the heart and cardiomyocytes. To test this, adult rat hearts were stimulated with Dex $10 \mathrm{nM}$ and we observed that $\mathrm{NO}$ production measured by quantification of nitrites was increased, but Dex did not activate iNOS measured by Western blot. Moreover, Dex did not induce an increase in the mRNA levels of iNOS in adult cardiomyocytes. Therefore, Dex generates NO independent of iNOS during its early pre-conditioning effect. Nevertheless, more studies are required to clarify its role in a possible long term protection against IR generated by Dex. Key Words: Dexmedetomidine, Heart, iNOS, pre-conditioning, ischemia-reperfussion. 


\section{Introducción}

El infarto del miocardio es una de las principales causas de muerte a nivel mundial, pudiendo ser un evento menor en una enfermedad crónica o manifestarse como un evento catastrófico que resulte en muerte súbita o en deterioro hemodinámico severo ${ }^{1}$.

El término infarto del miocardio refleja la muerte por necrosis de cardiomiocitos generada por una isquemia prolongada $^{2}$. En ese contexto, la reperfusión inmediata es de fundamental importancia para la viabilidad del miocardio. Sin embargo, este proceso a su vez desencadena la muerte celular a través de un mecanismo que implica la apertura del poro de transición de permeabilidad mitocondrial (PTPM), lo que se conoce como daño por reperfusión ${ }^{3}$.

El daño por isquemia del miocardio solía considerarse irreversible; sin embargo, en la actualidad se ha mostrado que al someter el tejido cardíaco a estrés por falta de oxígeno o al privarlo de sustratos, por periodos cortos, se activan mecanismos endógenos de sobrevida celular. Estos mecanismos moleculares favorecen condiciones fisiológicas de adaptación a la isquemia ${ }^{4}$. Los fenómenos de protección frente a un infarto del miocardio como consecuencia de breves períodos de isquemia previo o posterior a este proceso, se conocen como pre- y post-condicionamiento isquémico, respectivamente (PI y PostCI $)^{5-6}$. La forma clásica de PI temprano ocurre inmediatamente terminada la maniobra pre condicionante, sin embargo, también se ha descrito una segunda ventana de pre condicionamiento (PI tardío), que se lleva a cabo entre las 24 a 72 h y se relaciona con la expresión de genes que codifican para la expresión de proteínas de sobrevida celular ${ }^{7}$. En cuanto a los mecanismos que median los efectos del PI y PostCI se ha mostrado que durante la reperfusión se activan cascadas de señalización de proteínas pro-sobrevida entre las cuales destacan la fosfatidilinositol 3-kinasa (PI3K) y las proteínas kinasas AKT y ERK1/2. Esta vía se denomina RISK (Reperfusion Injury Salvage Kinases, por su sigla en inglés) ${ }^{8}$. Además, también se ha descrito la participación de otras proteínas como PKC- $\varepsilon$, PKG, p70S6K y GSK-3 $\beta$. La protección se deriva de la inhibición de la formación del PTPM a través de la activación de las kinasas de sobrevida AKT y ERK1/2, cuya inhibición del PTPM se ejerce por medio de la fosforilación de GSK-3 $\beta^{8,9}$.

Actualmente, también se ha establecido que estas vías de señalización celular protectoras se pueden activar mediante el uso de fármacos ${ }^{10}$. En ese contexto, dexmedetomidina (Dex) es un agonista altamente selectivo de los receptores alfa2-adrenérgicos que, además de su uso como sedante en anestesia11, ha sido descrito sólidamente como protector y pre-condicionante en múltiples modelos de isquemia-reperfusión (IR $)^{12-15}$. Asimismo, también se ha descrito su efecto protector en el corazón ${ }^{16-20}$; sin embargo, el mecanismo por el cual ejerce su efecto pre-condicionante aún está siendo estudiado.

Recientemente, en nuestro laboratorio se describió la participación del endotelio y la vía óxido nítrico sintasa endotelial (eNOS)-óxido nítrico (NO) en el pre-condicionamiento miocárdico con Dex (Fig 1) ${ }^{21}$. En el contexto de lo indicado, este trabajo mostró activación de eNOS y un aumento de NO en respuesta a Dex en corazón aislado de rata. Además, se observó que el efecto cardioprotector de Dex frente a IR se perdió al co-administrar el fármaco con PTIO (atrapador de NO) y L-NAME (inhibidor de NOS $)^{21}$. Sin embargo, cabe indicar que L-NAME es un inhibidor no específico de las $\mathrm{NOS}^{22}$, por lo que no se puede descartar la participación o efecto conjunto de las otras isoformas, nNOS e iNOS. La presente adenda tiene como objetivo evaluar si Dex activa a iNOS en el corazón y así evidenciar la posible participación de esta proteína en la cardioprotección generada por Dex.

\section{Materiales y Métodos}

\section{Reactivos}

El anticuerpo contra GAPDH, laminina y M-199 se obtuvieron en Sigma-Aldrich (St Louis, MO). Dexmedetomidina-HCl se obtuvo de Hospira, Chile. El anticuerpo contra iNOS se adquirió de BD Transduction Laboratories El kit colorimétrico para óxido nítrico se compró en Abcam (Cambridge, MA).

\section{Animales}

El presente estudio se ajustó a la Guía para el Cuidado y Uso de Animales de Laboratorio publicada por los Institutos Nacionales de Salud de Estados Unidos (8va Edición, 2011) y fue aprobado por el Comité de Ética Institucional. Las ratas se obtuvieron del Bioterio de la Facultad de Ciencias Químicas y Farmacéuticas, Universidad de Chile.

\section{Modelo ex vivo de corazón aislado}

De acuerdo al protocolo de perfusión retrograda con sistema Langendorff ${ }^{23}$, ratas Sprague-Dawley (250-350 g) se anestesiaron con pentobarbital ( $80 \mathrm{mg} / \mathrm{kg}$ i.p.). Posteriormente, se realizó una esternotomía y se administró heparina $100 \mathrm{U} / \mathrm{kg}$, i.v. Los corazones se extrajeron rápi- 
damente de la cavidad torácica, montados en una cámara de corazón con temperatura controlada y perfundidos de forma retrógrada a través de la aorta ascendente, con una solución tampón Krebs Henseleit que contenía (en mM) $\mathrm{NaCl}(128,3), \mathrm{KCl}(4,7), \mathrm{CaCl} 2(1,35), \mathrm{NaHCO} 3(20,2)$, NaH2PO4 $(0,4), \operatorname{MgSO} 4(1,1)$, glucosa $(11,1)$, a pH 7,4 a $37^{\circ} \mathrm{C}$, al equilibrarse con una mezcla de $95 \%$ O2 y $5 \% \mathrm{CO} 2$. Las temperaturas del perfusado se mantuvieron a $37^{\circ} \mathrm{C}$. Un balón de látex se insertó en el ventrículo izquierdo a través de la válvula mitral, se conectó a un transductor de presión (Puente Amp ADInstruments ML2 ${ }^{21}$, Australia) y se llenó con solución salina para producir una presión diastólica final del ventrículo izquierdo (PFD) de 5-10 mmHg. Los corazones se estabilizaron durante $20 \mathrm{~min}$, se pre-condicionaron con una infusión de Dex $10 \mathrm{nM}$ durante $25 \mathrm{~min}$ y, posteriormente, se realizó un lavado de 5 min con buffer Krebs-Henseleit. Luego, los corazones se congelaron a $-80^{\circ} \mathrm{C}$ para posterior análisis.

\section{Obtención de homogenizados de tejidos}

Los ventrículos de ratas adultas congelados en nitrógeno líquido se homogeneizaron en tampón frío con inhibidores de fosfatasas y proteasas (en $\mathrm{mM}$ ): MOPS-Tris $\mathrm{pH}$ $7^{20}$, Sacarosa (300), EDTA (2), EGTA (2) Na3V04 (10), NaF (80), Na4P2O7 (20), Nonidet P-40 del 1\% (v/v), SDS $0,1 \%(\mathrm{v} / \mathrm{v})$ y los inhibidores de proteasas leupeptina y pepstatina (1:1000), pH final 7,4. Luego las muestras se homogenizaron en Douncer vidrio-teflón y, posteriormente, se centrifugaron a $1000 \mathrm{x}$ g durante $20 \mathrm{~min}$ a $4^{\circ} \mathrm{C}$. Posteriormente, se determinó la concentración de proteínas por el método de Hartree ${ }^{24}$. Las fracciones solubles se guardaron congeladas a $-80^{\circ} \mathrm{C}$, para posterior análisis por Western blot.

\section{Western blot y determinación de dimeros de iNOS:}

La dimerización de iNOS se evaluó utilizando electroforesis de baja temperatura (LT SDS PAGE) según la técnica descrita ${ }^{25}$. Los niveles de la forma dimerizada de iNOS en los ventrículos de ratas adultas se determinaron por Western blot, al final del periodo de precondicionamiento farmacológico. Para la electroforesis y el análisis de Western blot, los lisados de los tejidos se ajustaron por proteínas $(20 \mu \mathrm{g})$, separadas por LT-SDS-PAGE (8\%). Los geles se mantuvieron previamente a $4^{\circ} \mathrm{C}$ y la cámara de electroforesis se mantuvo en una fuente con hielo, de manera tal que se mantuviese la temperatura del gel $\left(<10^{\circ} \mathrm{C}\right)$ hasta ser transferidos a membranas de PVDF. Posteriormente, las membranas se bloquearon con BSA al $5 \% \mathrm{p} / \mathrm{v}$ en TBS Tween $0,2 \%$ por $1 \mathrm{~h}$. Las membranas se incubaron con anticuerpos contra la forma total (1:1.000) de iNOS, así como también un anticuerpo contra GAPDH como control de carga (1:20.000). Después de un período de incubación adicional con los correspondientes anticuerpos secundarios anti IgG de conejo o ratón, conjugado a peroxidasa de rabanito (1:5.000), la unión específica se detectó mediante quimioluminiscencia (ECL). La intensidad de la señal de las bandas se cuantificó por densitometría usando el software Image J.

\section{Determinación de nitritos por reacción de Griess}

La concentración de nitritos se cuantificó por medio de la reacción de Griess, utilizando el kit ab65328 Nitric Oxide Assay Kit Colorimetric (Abcam). Las muestras se obtuvieron en el efluente coronario posterior a los $5 \mathrm{~min}$ de lavado que suceden el tratamiento con o sin Dex $10 \mathrm{nM}$.

\section{Cultivo primario de cardiomiocitos adultos de rata}

Ratas adultas Sprague-Dawley (250-350 g) se anestesiaron con pentobarbital ( $80 \mathrm{mg} / \mathrm{kg}$ IP), y los corazones se aislaron y perfundieron de forma retrograda a través de la

\section{Figura 1:}

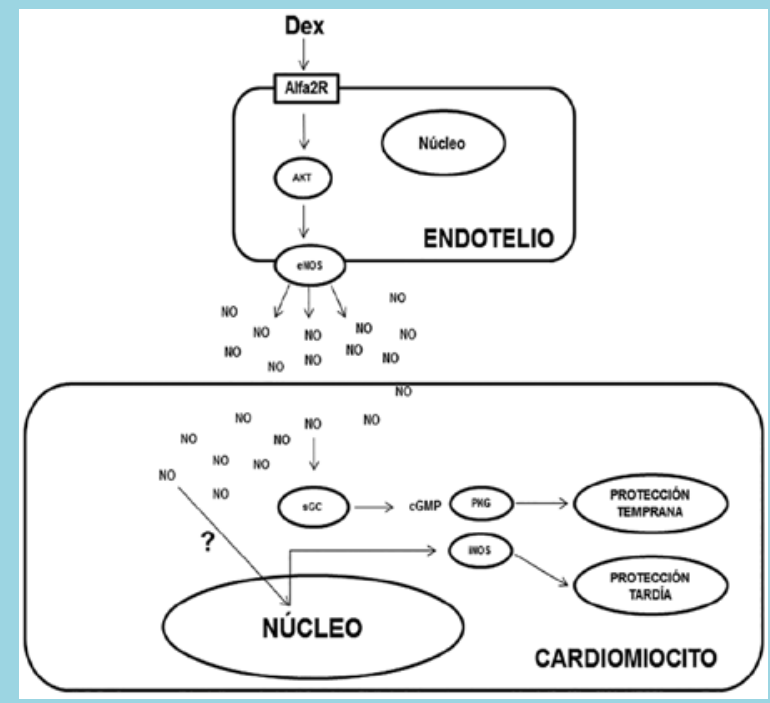

Fig. 1. Dex protege frente a IR a través de un mecanismo dependiente del endotelio y la vía eNOS NO. Dex se une al receptor alfa2-adrenérgico (Alfa2R) en el endotelio, promoviendo la activación de AKT, que a su vez activa a la óxido nítrico sintasa endotelial (eNOS). El óxido nítrico (NO) generado por eNOS difunde desde el endotelio hacia el cardiomiocito, activando a la guanilato ciclasa soluble (sGC), la cual produce GMP cíclico (cGMP), que activa la proteína kinasa dependiente de cGMP(PKG), generando así la protección temprana del miocardio. Asimismo, el NO puede promover protección tardía, la cual podría estar mediada por la inducción de la óxido nítrico sintasa inducible (iNOS). 
aorta. Los cardiomiocitos se aislaron administrando una infusión de colagenasa tipo II, según la técnica descrita ${ }^{26}$. Luego, las células se sembraron en placas con laminina e incubadas durante $e^{6-24} \mathrm{~h}$ a $37^{\circ} \mathrm{C}$ en condiciones de $95 \%$ oxígeno y $5 \% \mathrm{CO} 2$. Las células se mantuvieron en medio M-199 (M7653, Sigma) suplementado con 10\% de suero fetal de ternero y $1 \%$ penicilina - estreptomicina (Sigma).

\section{PCR a tiempo real (qPCR)}

Cardiomiocitos de rata adulta se estimularon con Dex 10 nM durante $0.5,1,2$ y 3 h. PCR a tiempo real se realizó utilizando SYBR Green (Applied Biosystems), según el protocolo ya descrito ${ }^{27}$. Los datos obtenidos para cada transcrito se normalizaron respecto al rRNA $18 \mathrm{~S}$ como control interno, usando el método $2-\Delta \Delta \mathrm{Ct}$. Los partidores utilizados fueron los siguientes:

\section{-iNOS (rata) sentido TTGGTACATGGGCACCGAGATTG} - iNOS (rata) antisentido CAGCTGCATTGATCTCGGTGACA

\section{Análisis estadístico}

Los resultados se muestran como el promedio \pm SEM de al menos 3 experimentos independientes. Los datos se analizaron utilizando t test o ANOVA de una vía. Las diferencias se consideraron significativas con $\mathrm{p}<0.05$.

\section{Resultados y Discusión}

Para evaluar si Dex activa a iNOS en el miocardio, corazones aislados de rata adulta se estimularon durante $25 \mathrm{~min}$ con Dex $10 \mathrm{nM}$ y posteriormente se generó un lavado de 5 min con buffer Krebs-Henseleit. Posterior a este lavado, se midió la generación de nitritos, que son un marcador de $\mathrm{NO}^{28}$ y se observó que Dex aumentó su producción (Fig 2A), lo cual concuerda con trabajos anteriores que describen la capacidad de Dex de generar $\mathrm{NO}^{21-29-30}$. Sin embargo, al evaluar la formación de dímeros (estado activo de la isoforma inducible de $\mathrm{NOS}^{31}$ ), no se observaron cambios respecto al control (Fig 2B). Para confirmar estos resultados se evaluaron los niveles de mRNA de iNOS en cardiomiocitos de rata adulta en respuesta Dex $10 \mathrm{nM}$ y no se observaron cambios respecto al control (Fig 2C). Estos resultados apoyan los hallazgos que sugieren que iNOS se asocia a la segunda ventana de cardioprotección o PI tardío ${ }^{32}$ y no al PI agudo. Sin embargo, en ese mismo sentido, no se puede descartar una posible participación de iNOS en la protección generada con Dex a largo plazo, ya que este fármaco genera NO, el cual se ha descrito como uno de los estímulos que gatilla el PI tardío ${ }^{33}$ y por

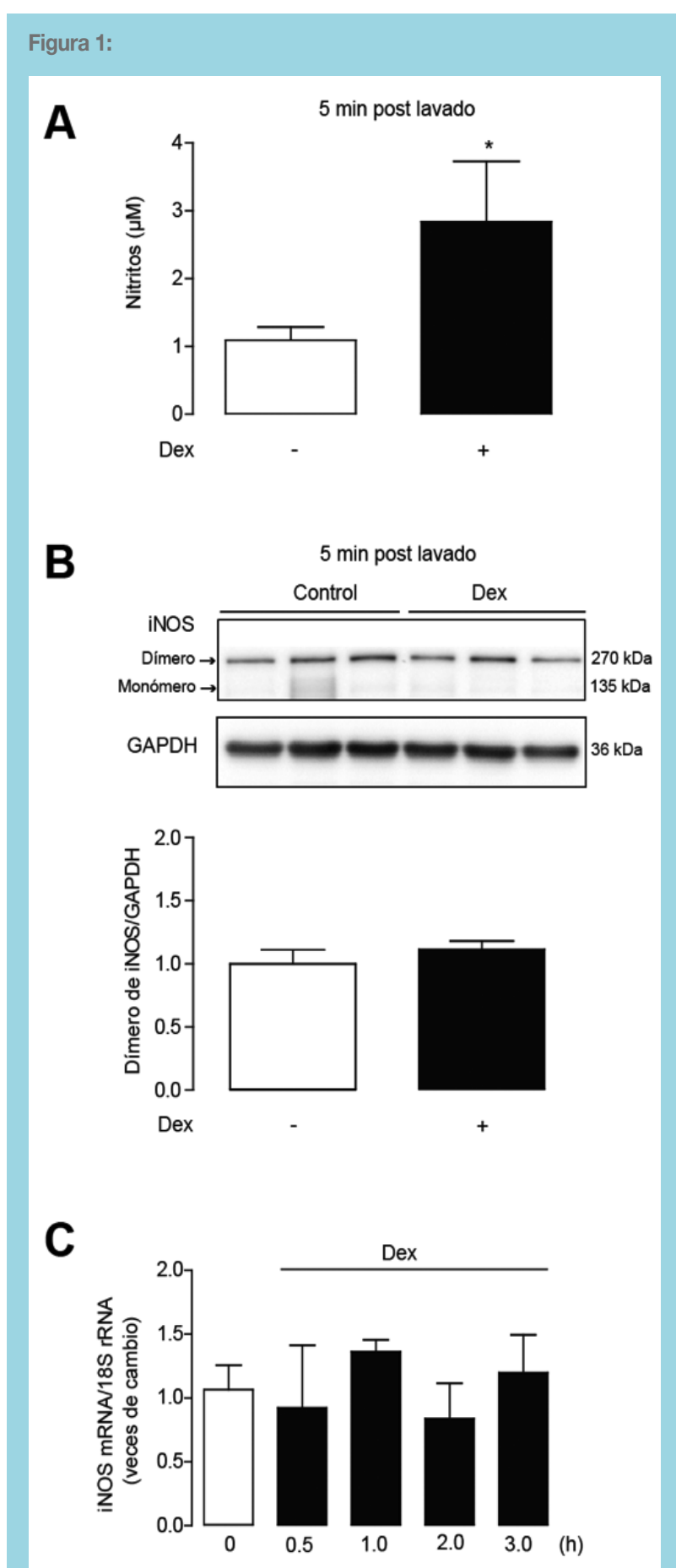

Fig. 2. Dex aumenta la producción de nitritos pero no la dimerización o niveles de mRNA de iNOS. (A): Concentración de nitritos en corazones aislados de rata luego de 5 min de lavado posterior a pre-condicionamiento con o $\sin$ Dex $10 \mathrm{nM}$.(B) Panel superior: Western blot representativo de dímeros de iNOS en homogenizados de corazón. En el panel inferior se muestra la cuantificación de los dímeros de iNOS normalizados por GAPDH. (C) Niveles de mRNA de iNOS en cardiomiocitos de rata adulta estimulados con Dex $10 \mathrm{nM}$ durante $0.5,1,2, y 3 \mathrm{~h}$. Las barras representan el promedio + SEM de 3 determinaciones. $* P<0,05$ vs Control. Los datos se analizaron por $t$ test pareada con una cola, seguido por post-test de Mann-Whitney. 


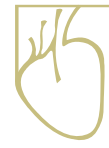

lo tanto, podría inducir la expresión de iNOS (Fig 1). Por lo tanto, se requieren estudios de IR in vivo en los cuales se determinen los niveles y activación de iNOS 24 y 72 $\mathrm{h}$ en corazones estimulados con o sin Dex. Asimismo, estos hallazgos podrían ser reforzados con estudios de IR in vitro, utilizando siRNAs para nNOS e iNOS, con el fin de descartar con precisión la participación de estas isoformas de NOS.

En resumen, Dex protege el miocardio del daño agudo por IR a través de un mecanismo que involucra el endotelio y la vía eNOS-NO, independiente de iNOS. Sin embargo, la isoforma inducible pudiese participar en la protección con Dex a largo plazo y futuros estudios deben clarificar este punto. Asimismo, Dex es un fármaco cuyo efecto cardioprotector se encuentra ampliamente documentado,
Dexmedetomidina genera óxido nítrico mediante un mecanismo... Riquelme J., et al.

pero los estudios que detallen su mecanismo de acción en este pre condicionamiento no son menos importantes, ya que un correcto y detallado entendimiento de la señalización celular generada por Dex tanto en la primera como en la segunda ventana de protección, asegurará su uso seguro y permitirá a su vez, desarrollar nuevas estrategias de protección frente al infarto del miocardio.

\section{Agradecimientos}

Este trabajo se financió a través del Proyectos FONDECYT (3160298 para J.A.R., 3140532 para F.W., 1130407 para G.S., 1150887 para Z.P.), y CONICYT (FONDAP 15130011 para S.L.). Los autores agradecen a G. Arce, R. Durán, F. Albornoz, G. Latorre y L. Montecinos por su excelente apoyo técnico. 


\section{Referencias}

1 THYGESEN K, ALPERT JS, WHITE HD, JOINT ESCAAHAWHFTFFTROMI, JAFFE AS, APPLE FS, et al. Universal definition of myocardial infarction. Circulation 2007;116:2634-2653.

2 ALPERT JS, THYGESEN K, ANTMAN E, BASSAND JP. Myocardial infarction redefined--a consensus document of the joint european society of cardiology/american college of cardiology committee for the redefinition of myocardial infarction. J Am Coll Cardiol 2000;36:959-969.

3 HAUSENLOY DJ, YELLON DM. Ischemic conditioning and reperfusion injury. Nature Rev Cardiol 2016

4 DEPRE C, VATNER SF. Cardioprotection in stunned and hibernating myocardium. Heart Fail Rev 2007;12:307-317.

5 MURRY CE, JENNINGS RB, REIMER KA. Preconditioning with ischemia: A delay of lethal cell injury in ischemic myocardium. Circulation 1986;74:1124-1136.

6 ZHAO ZQ, CORVERA JS, HALKOS ME, KERENDI F, WANG NP, GUYTON RA, et al. Inhibition of myocardial injury by ischemic postconditioning during reperfusion: Comparison with ischemic preconditioning. Am J Physiol Heart Circ Physiol 2003;285:H579-588.

7 BAXTER GF, YELLON DM. Ischaemic preconditioning of myocardium: A new paradigm for clinical cardioprotection? British J Clin Pharmacol 1994;38:381-387.

8 HAUSENLOY DJ, YELLON DM. Reperfusion injury salvage kinase signalling: Taking a risk for cardioprotection. Heart Fail $\operatorname{Rev}$ 2007;12:217-234.

9 DOWNEY JM, DAVIS AM, COHEN MV. Signaling pathways in ischemic preconditioning. Heart Fail Rev 2007;12:181-188.

10 SIVARAMAN V, YELLON DM. Pharmacologic therapy that simulates conditioning for cardiac ischemic/reperfusion injury. J Cardiovasc Pharmacol Ther 2014;19:83-96.

11 BHANA N, GOA KL, MCCLELLAN KJ. Dexmedetomidine. Drugs 2000;59:263-268.

12 ZHU YM, WANG CC, CHEN L, QIAN LB, MA LL, YU J, et al. Both pi3k/akt and erk $1 / 2$ pathways participate in the protection by dexmedetomidine against transient focal cerebral ischemia/reperfusion injury in rats. Brain Res 2013;1494:1-8.

13 LEMPIAINEN J, FINCKENBERG P, MERVAALA EE, STORVIK M, KAIVOLA J, LINDSTEDT K, et al. Dexmedetomidine preconditioning ameliorates kidney ischemia-reperfusion injury. Pharmacol Res Persp 2014;2:e00045

14 JIANG L, LI L, SHEN J, QI Z, GUO L. Effect of dexmedetomidine on lung ischemiareperfusion injury. Mol Med Rep 2014;9:419-426.

15 KUCUK A, YAYLAK F, CAVUNT-BAYRAKTAR A, TOSUN M, ARSLAN M, COMU FM, KAVUTCU M. The protective effects of dexmedetomidine on hepatic ischemia reperfusion injury. Bratislavske lekarske listy 2014;115:680-684.

16 JIANG C, XIA M, WANG M, CHEN S. [dexmedetomidine preconditioning protects isolated rat hearts against ischemia/ reperfusion injuries and its mechanism]. J Zhejiang Univ Med Sci 2013;42:326-330.

17 IBACACHE M, SANCHEZ G, PEDROZO Z, GALVEZ F, HUMERES C, ECHEVARRIA G, DUASO J, HASSI M, GARCIA L, DIAZ-ARAYA G, LAVANDERO S. Dexmedetomidine preconditioning activates pro-survival kinases and attenuates regional ischemia/reperfusion injury in rat heart. Biochim Biophys Acta- Mol Basis Dis 2012;1822:537-545.

18 OKADA H, KURITA T, MOCHIZUKI T, MORITA K, SATO $\mathrm{S}$. The cardioprotective effect of dexmedetomidine on global ischaemia in isolated rat hearts. Resuscitation 2007;74:538545.

19 YOSHITOMI O, CHO S, HARA T, SHIBATA I, MAEKAWA T, URESHINO H, SUMIKAWA K. Direct protective effects of dexmedetomidine against myocardial ischemia-reperfusion injury in anesthetized pigs. Shock 2012;38:92-97.

20 KOCOGLU H, KARAASLAN K, GONCA E, BOZDOGAN O, GULCU N. Preconditionin effects of dexmedetomidine on myocardial ischemia/reperfusion injury in rats. Curr Therap Res Clin Exp. 2008;69:150-158.

21 RIQUELME JA, WESTERMEIER F, HALL AR, VICEN- 
CIO JM, PEDROZO Z, IBACACHE M, FUENZALIDA B, SOBREVIA L, DAVIDSON SM, Yellon DM, Sanchez G, Lavandero S. Dexmedetomidine protects the heart against ischemia-reperfusion injury by an endothelial enos/no dependent mechanism. Pharmacol Res 2015;103:318-327.

22 STEVANOVIC ID, JOVANOvic MD, Jelenkovic A, Colic M, Stojanovic I, Ninkovic M. Effects of 1-name, a non-specific nitric oxide synthase inhibitor, on alc13-induced toxicity in the rat forebrain cortex. J Vet Sci 2009;10:15-22.

23 BELL RM, MOCANU MM, YELLON DM. Retrograde heart perfusion: The langendorff technique of isolated heart perfusion. J Mol Cell Cardiol 2011;50:940-950.

24 HARTREE EF. Determination of protein: A modification of the lowry method that gives a linear photometric response. Anal Biochem 1972;48:422-427.

25 CHUNG BH, KIM S, KIM JD, LEE JJ, BAEK YY, JEOUNG D, LEE H, CHOE J, HA KS, WON MH, KWON YG, KIM YM. Syringaresinol causes vasorelaxation by elevating nitric oxide production through the phosphorylation and dimerization of endothelial nitric oxide synthase. Exp Mol Med 2012;44:191-201.

26 HAUSENLOY DJ, YELLON DM, MANI-BABU S, DUCHEN MR. Preconditioning protects by inhibiting the mitochondrial permeability transition. Am J Physiol Heart Circ Physiol 2004;287:H841-849.

27 GUTIERREZ T, PARRA V, TRONCOSO R, PENNANEN C, CONTRERAS-FERRAT A, VASQUEZ-TRINCADO C, MORALES PE, LOPEZ-CRISOSTO C, SOTOMAYOR-FLORES
C, CHIONG M, ROTHERMEL BA, LAVANDERO S. Alteration in mitochondrial $\mathrm{ca}(2+)$ uptake disrupts insulin signaling in hypertrophic cardiomyocytes. Cell Commun Signal 2014;12:68

28 KELM M. Nitric oxide metabolism and breakdown. Biochim Biophys Acta 1999;1411:273-289.

29 KIM HJ, SOHN JT, JEONG YS, CHO MS, KIM HJ, CHANG KC, SHIN MK, PARK CS, CHUNG YK. Direct effect of dexmedetomidine on rat isolated aorta involves endothelial nitric oxide synthesis and activation of the lipoxygenase pathway. Clin Exp Pharmacol Physiol 2009;36:406-412.

30 JOSHI MS, FERGUSON TB, JR., JOHNSON FK, JOHNSON RA, PARTHASARATHY S, LANCASTER JR, JR. Receptor-mediated activation of nitric oxide synthesis by arginine in endothelial cells. Proc Natl Acad Sci USA 2007;104:9982-9987.

31 RAFIKOV R, FONSECA FV, KUMAR S, PARDO D, DARRAGH C, ELMS S, FULTON D, BLACK SM. Enos activation and no function: Structural motifs responsible for the posttranslational control of endothelial nitric oxide synthase activity. J Endocrinol 2011;210:271-284.

32 GUO Y, JONES WK, XUAN YT, TANG XL, BAO W, WU WJ, HAN H, LAUBACH VE, PING P, YANG Z, QIU Y, BOLLI $R$. The late phase of ischemic preconditioning is abrogated by targeted disruption of the inducible no synthase gene. Proc Natl Acad Sci USA 1999;96:11507-11512.

33 BOLLI R. The late phase of preconditioning. Circ Res 2000;87:972-983. 